\title{
Digital medicine
}

Al-facilitated health care requires education of clinicians

Artificial intelligence (AI) and deep learning have changed our lives. In our smartphones, laptops, and social media, they provide image and speech recognition, language translation, and more. These advances have begun to percolate into medicine, with deep learning systems capable of diagnosing skin cancer and fully autonomous Al approved for diabetic retinopathy screening. Although Al-enabled health care has huge potential, we are still only in its early stages, with much work to be done before its benefits can truly be achieved. As the field matures, a key question is to how should clinicians be educated in these advances? And what role should they play in developing, validating, and implementing these technologies?

Consider a thought experiment: in 2016 , as the tech industry was being transformed by Al, what was required to develop a deep learning system in a clinical setting? Technical expertise was fundamental, but limited to a fairly small number of practitioners. Access to substantial computing resources, particularly graphics processing units (GPUs), was also a prerequisite. And lastly, large datasets, often running to millions of images, were vital. Addressing these needs was challenging; many of experts in this field were quickly hired by multinational tech companies; there was little if any access to GPUs in hospital settings, where most large clinical datasets exist; and access to large datasets was extremely limited, partly due to privacy concerns. So how have things changed in 2021? Flexible, scalable, computing resources are now available on tap through cloud-based infrastructure, something which health systems have begun to adopt, while the use of privacy protecting techniques, such as [blockchain-based?] federated learning, offer the potential for the first truly global datasets. Perhaps most importantly, deep learning has become easier with the open sourcing of software libraries and the advent of so-called automated deep learning.

Automated deep learning systems provide intuitive, drag-and-drop interfaces that allow users to develop deep learning models with minimal, if any, coding. In 2019 , the feasibility of using one such system was shown for multiple clinical applications-eg, chest x-rays, skin lesions, retinal photographs. Research involving state-of-the-art models have recently been replicated using code-free deep learning. In the coming years, these systems are expected to be used to replicateor surpass-other major breakthroughs in clinical Al. As their potential develops, it seems likely that automated deep

learning systems will have a central role in medical education and subsequently the democratisation of Al development for health care.

In the initial feasibility study from 2019, clinicians quickly became facile with automated deep learning for various medical imaging applications. This is one dimension of how health-care professionals can and need to be educated about the potential utility and the nuances of Al. It will be essential for any important matter in health care that there is a "human-in-the-loop" and this clinician will need to be familiar with the shortcomings and strengths of a deep neural network's output. With automated deep learning platforms, it will be possible for clinicians to explore this using publicly available medical datasets that do not require any additional ethical or institutional approvals. Beyond this direct experience, multiple online courses have been initiated to train physicians and nurses on the basics of Al and its applications for health care, including how to critically appraise the literature. It will soon be incumbent on medical schools to update their curricula to include deep and machine learning so the future workforce will be well equipped to integrate $\mathrm{Al}$ into patient care as the field advances. 
The Al revolution is approaching a pivot point, perhaps akin to that reached by the computer industry in the late 1970 s. At that time, the industry was focused on mainframe computers, typically provided to large corporations, universities, or the military, for applications such as payroll calculation and rocket design. It's hard to imagine now, but at that time the idea of personal computers was strange and unlikely. But a group of hobbyists and hackers spotted that, although initially primitive, personal computers would lead to countless, hitherto unimagined applications. We believe we are approaching a new phase for Al-enabled health care that is akin to what happened with computing. Clinicians will have an increasingly important role in this Al revolution that will ultimately lead to countless benefits for our patients. However, for Al-enabled health care to be actualised, it is time to develop broad educational initiatives for the medical community.

*Pearse Keane, Eric Topol

Moorfields Eye Hospital NHS Foundation Trust, London EC1V 2PD, UK (PK); and Scripps Research Translational Institute, La Jolla, CA, USA (ET)

pearse.keane1@nhs.net

PAK is by a Moorfields Eye Charity Career Development Award (R190028A) and a UK Research \& Innovation Future Leaders Fellowship (MR/T019050/1).

EJT is supported by the US National Institutes of Health/National Center for Advancing Translational Sciences grant UL1TR001114.

\section{Further Reading:}

Lewis-Kraus G. The Great A.I. Awakening. The New York Times, Dec $14^{\text {th }} 2016$

https://www.nytimes.com/2016/12/14/magazine/the-great-ai-awakening.html (accessed March 10th 2021)

Faes L. et al. Automated deep learning design for medical image classification by health-care professionals with no coding experience: a feasibility study. The Lancet Digital Health 1, e232-e242 (2019).

Korot E, et al. Code-free Deep Learning for Medical Image Analysis: A Multi-modality Assessment of Platforms. Nature Machine Intelligence (2021).

Khan SM et al. A global review of publicly available datasets for ophthalmological imaging: barriers to access, usability, and generalisability. The Lancet Digital Health (2020) doi:10.1016/s2589-7500(20)30240-5.

American College of Radiology (ACR) Al-Lab. https://ailab.acr.org/Account/Login (accessed March 10th 2021). 
Liu, Y., Chen, P.-H. C., Krause, J. \& Peng, L. How to Read Articles That Use Machine Learning. Jama 322, 1806 (2019).

Faes, L. et al. A Clinician's Guide to Artificial Intelligence: How to Critically Appraise Machine Learning Studies. Trans/ Vis Sci Technology 9, 7 (2020). 